\title{
Voluntary counselling and testing reduced unprotected intercourse among adults in 3 developing countries
}

The Voluntary HIV-1 Counseling and Testing Efficacy Study Group. Efficacy of voluntary HIV-1 counselling and testing in individuals and couples in Kenya, Tanzania, and Trinidad: a randomised trial. Lancet 2000 Jul 8;356:103-12.

\section{QUESTION: In adults in developing countries, is a voluntary counselling and testing (VCT) programme as effective as a health information programme in reducing risk behaviour associated with sexual transmission of HIV-1?}

\section{Design}

Randomised (allocation concealment unclear*), unblinded,* controlled trial with mean 14 months follow up.

\section{Setting}

Nairobi, Kenya; Dar es Salaam, Tanzania; and Port of Spain, Trinidad.

\section{Participants}

4293 participants (3120 as individuals and 1173 as couples) who were $\geqslant 18$ years of age (mean age $29 \mathrm{y}$ ) and were not known to be infected with HIV-1. First follow up data at a mean of 7.3 months were available for 2550 participants as individuals (82\%) and for 1001 as couples $(85 \%)$.

\section{Intervention}

Participants were stratified by site, sex, and couple or individual status and allocated to VCT $(\mathrm{n}=1563,589$ as couples) or health information ( $\mathrm{n}=1557,584$ as couples). VCT involved personalised risk assessment, development of a plan for risk reduction with a counsellor, and ELISA testing of serum samples for HIV-1. The control intervention involved watching a 15 minute video and participating in a group discussion led by a health information officer about HIV-1 transmission and condom use. Participants in both groups received 25 condoms and a brochure showing correct condom use and could return any time for more condoms. All participants engaged in a baseline interview to assess HIV-1 risk behaviour.

\section{Main outcome measure}

Rate of unprotected intercourse assessed during follow up interviews.

Sources of funding: US

Agency for

International

Development; World

Health Organisation;

United Nations

Programme on AIDS

National Institute of

Mental Health.

For correspondence: Dr T J Coates, Center for AIDS Prevention Studies, University of California, San

Francisco, 74 New

Montgomery \#600,

San Francisco, $C A$

94105, USA. Fax +1

4155979213.

\section{Main results}

Between baseline and the first follow up, rates of unprotected intercourse with non-primary partners decreased more in participants who received VCT than in those who received the health information control intervention (for men: relative rate reduction $35 \%$ v $13 \%$, $\mathrm{p}=0.01$; for women: relative rate reduction $39 \% v 17 \%$, $\mathrm{p}=0.009$ ). Among couples, men in the VCT group reported a greater reduction in rates of unprotected intercourse with enrolment partners than men in the control group (relative rate reduction 25\% v 15\%, $p=0.008$ ), but rates of unprotected intercourse with non-enrolment partners were not reduced. Rates of unprotected intercourse with non-primary partners decreased more in HIV infected men than in uninfected men; among HIV infected women, rates with primary partners decreased.

\section{Conclusion}

In adults in developing countries, a voluntary counselling and testing programme was more effective than a health information programme in reducing risk behaviour associated with sexual transmission of HIV-1 at a mean of 7.3 months of follow up.

*See glossary.

\section{COMMENTARY}

In the absence of a suitable vaccine for HIV control, attempts to alter and thus reduce high risk behaviour remain a primary effort for physicians and patients. The study by the Voluntary HIV-1 Counseling and Testing Efficacy Study Group selected patients from 3 developing countries to test whether counselling and free condoms reduce rates of infection more than standard health information dissemination. Data are dependent on patient recall of unprotected sex (non-condom) and follow up serological findings for case confirmation.

Although rate reductions of unprotected intercourse with VCT seem reasonable, the nature of HIV-1 infection means that this intervention could only be expected to make a small contribution to the efforts to control or eliminate the disease in a community. Even if no errors occurred in the data recall set or during sampling or date recording, the failure to affect the sexual behaviour practices of $\geqslant 60 \%$ of people suggests that with a reservoir of HIV infection in the community the rates of infection are unlikely to differ after 1 year. This would be especially true if free condoms were no longer available and the counselling services were stopped. The study sample size is large, but it pales when compared with total populations at risk in developing countries, where most estimates of current prevalence range from about $1 \%$ to $13 \%$ but are higher in some countries. ${ }^{1}$ These rates will increase as illicit drug use becomes more prevalent and as economic and cultural development ensues.

Individual physicians may take comfort in risk reductions in some of their patients as a result of the counselling techniques described here, but they must be prepared for longer term follow up, the inevitable failures to reduce incidence, and the consequences of condom cost and use. This study is commendable for its methods and data analysis, but attrition rate and behavioural change failure rate detract from its effectiveness. Such community based programmes may continue to be used, however, pending an effective vaccine and vaccination programme.

Edward A Lichter, MD University of Illinois College of Medicine Chicago, Illinois, USA

1 Joint United Nations Programme on HIV/AIDS (UNAIDS) Report on the global HIV/AIDS epidemic, June 2000. (http:// www.unaids.org/epidemic_update/report/). 